\title{
Molecular Characterization of a New G (VP7) Genotype in Group B Porcine Rotavirus
}

\author{
Bruna Letícia Domingues Molinari ${ }^{a, c}$ Alice Fernandes Alfieri ${ }^{a, b}$ \\ Amauri Alcindo Alfieri ${ }^{a, b}$ \\ a Laboratory of Animal Virology, Department of Veterinary Preventive Medicine, Universidade Estadual de Londrina, \\ Londrina, Brazil; b Multi-User Animal Health Laboratory - Molecular Biology Unit, Department of Veterinary \\ Preventive Medicine, Universidade Estadual de Londrina, Londrina, Brazil; ' Laboratory of Clinical Veterinary \\ Microbiology, UNINGA - Centro Universitário Ingá, Maringá, Brazil
}

\author{
Keywords \\ Diarrhea outbreak · Novel G genotype · Pigs · Reverse \\ transcription PCR $\cdot$ Rotavirus group $B$
}

\begin{abstract}
Rotaviruses (RVs), a common cause of viral gastroenteritis in humans and animals, are classified into 9 established groups/ species (RVA-RVI). Although RVB has been found in several countries, genetic variation among RVB field strains remains poorly characterized. RVB strains can be classified into G genotypes based on a nucleotide (nt) homology that exceeds a cutoff value of $80 \%$ for the gene that encodes the structural protein VP7. In this study, we determined the VP7 nt and deduced amino acid sequences of one RVB strain (RB62) identified in a diarrheic fecal sample obtained from a piglet in Brazil in 2012. Comparative analysis of this strain and the strains of the other 21 previously identified VP7 genotypes showed that the highest nt identity (71.2\%) was found with the porcine PB-70-H5 strain within the G4 genotype. However, when compared with the nonclassified Vietnamese RVB G genotype 14177_18 strain, the nt sequence
\end{abstract}

identity was of $82.9 \%$. These results led us to conclude that the Brazilian strain BR62 and the Vietnamese strain 14177_18 belong to a novel G genotype (G22).

(c) 2018 S. Karger AG, Basel

Rotaviruses (RVs), which are members of the Reoviridae family, are a common cause of viral gastroenteritis in humans and animals. RVs consist of nonenveloped particles and possess a genome composed of 11 segments of double-stranded RNA that encode 6 structural (VP1VP4, VP6, and VP7) and 6 nonstructural (NSP1-NSP5/6) proteins [1].

RVs are classified into 9 established groups/species (RVA-RVI) based on genetic and antigenic differences in the structural VP6 protein [2-4]. RV strains from groups $\mathrm{A}, \mathrm{B}, \mathrm{C}$, and $\mathrm{H}$ are known to infect humans and various animal species, whereas strains from the other RV groups have been found to infect only animals [3-5].

Among animals, RVB has been found in pigs, cattle, goats, and rats [1]. However, in contrast to RVA, porcine RVB infections have been sporadically reported in pig

\section{KARGER}

(c) 2018 S. Karger AG, Basel

E-Mail karger@karger.com

www.karger.com/int
Amauri Alcindo Alfieri, $\mathrm{MS}_{\mathrm{c}}, \mathrm{PhD}$

Laboratory of Animal Virology, Department of Veterinary Preventive Medicine Universidade Estadual de Londrina, P.O. Box 10011

Londrina, Paraná 86057-970 (Brazil)

E-Mail alfieri@uel.br 
herds in the UK [6], Australia [7], New Zealand [8], Japan [9], the Czech Republic [10], Brazil [11], the USA [12], and Vietnam [13]. The relatively few worldwide reports of RVB infection in animals may be attributed to the fact that small quantities of this virus are excreted in the feces of infected animals, which affects the diagnosis of RVB infections $[9,12,14]$.

Although RVB has been found in several countries, genetic variation among RVB strains remains poorly characterized. Knowledge regarding the genome of porcine RVB remains restricted to information obtained via analyses of the VP6, VP7, NSP1, NSP2, and NSP5 proteins $[3,9,11,12,14-16]$. Similar to RVA strains, RVB strains are categorized using a sequence-based classification system $[12,16,17]$. RVB strains can be classified into G genotypes based on a nucleotide (nt) cutoff value of $80 \%$ for the gene that encodes the structural protein VP7 [17]. To our knowledge, 21 RVB G genotypes have previously been described $[13,17]$.

In this study, we determined the VP7 nt and deduced amino acid (aa) sequences of one RVB strain identified in a diarrheic fecal sample obtained from a piglet in Brazil in 2012. Comparative analysis of this strain and the strains of the other 21 previously identified VP7 genotypes showed that the Brazilian porcine RVB strain belongs to a novel VP7 genotype, G22, and seems to be circulating in different parts of the world.

A total of 50 diarrheic fecal samples, collected from pigs 28 and 35 days of age, from a post-weaning diarrhea outbreak that occurred in a pig herd located in Mato Grosso do Sul, Brazil, in 2012, were subjected to reverse transcription PCR to investigate the presence of RV groups A, B, C, and H. According to Molinari et al. [18], all samples were tested for RVB using the primer pair described by Marthaler et al. [12], which was designed to amplify a fragment (778 bp) of the VP7 gene of RVB. Sixteen diarrheic fecal samples were positive for RVB [19]. Based on the quality of the amplified product, the RVBpositive fecal sample identified as BR62 was selected for further molecular analyses.

To confirm the specificity of the RVB amplicon, the amplified product was purified using a GFX ${ }^{\mathrm{TM}}$ PCR DNA and Gel Band Purification Kit (GE Healthcare, Little Chalfont, UK), quantified on a Qubit ${ }^{\mathrm{TM}}$ Fluorometer (Invitrogen - Life Technologies, Eugene, OR, USA), and sequenced using a BigDye Terminator v3.1 Cycle Sequencing Reaction Kit (Applied Biosystems, Foster City, CA, USA) on an automated sequencer (ABI3500; Applied Biosystems). Sequence quality analyses were performed using the Phred and CAP3 software packages (http://as- pargin.cenargen.embrapa.br/phph/). Similarity searches were performed against sequences deposited in GenBank using the Basic Local Alignment Search Tool (BLAST) (http://blast.ncbi.nlm.nih.gov/Blast.egi). Phylogenetic trees based on nt sequences were constructed using the neighbor joining method from the Kimura two-parameter model, which provided statistical support via bootstrapping with 1,000 replicates in the MEGA software package (version 6). $\mathrm{Nt}$ and aa sequence identity matrices were constructed using BioEdit software version 7.08.0. The nt sequence for the VP7 gene of the BR62 RVB strain described in this study was deposited in the GenBank database under accession No. MF072691.

A comparative sequence analysis involving the VP7 genes of the Brazilian RVB strain BR62 and strains belonging to the 21 known RVB G genotypes was performed. Additionally, the VP7 gene of the unclassified porcine RVB strain 14177_18, recently described in Vietnam, was included in this analysis [20].

Compared with the strains belonging to the 21 established RVB G genotypes, the Brazilian wild-type porcine RVB strain BR62 showed the highest nt identity (71.2\%) with the porcine PB-70-H5 strain within the G4 genotype. The lowest nt identity (54.6\%) was observed with the IDIR strain within the G1 genotype. Pairwise comparisons with the other genotypes showed similarities ranging from 61.8 to $70.9 \%$ at the nt level (Table 1). In contrast, a comparison of the BR62 strain and the Vietnamese 14177_18 strain revealed an nt sequence identity of $82.9 \%$.

A phylogenetic analysis based on deduced aa sequences for RVB VP7 was also performed. This analysis indicated that the BR62 strain showed the highest aa identity (88.7\%) with the Vietnamese 14177_18 strain. The BR62 strain's aa identity with other RVB strains ranged from 48.6 to $75.9 \%$ (Table 1 ).

In phylogenetic trees based on VP7 nt and aa sequences for VP7, the porcine field strain BR62 described in this study constituted a branch separate from the strains belonging to the 21 previously identified RVB genotypes. Additionally, the unclassified Vietnamese 14177_18 strain was clustered with the BR62 strain (Fig. 1).

Although less prevalent than RVA infections, RVB infections in humans and pigs have been reported in different regions throughout the world [19, 20-22]. However, small quantities of this virus are excreted in the feces of infected animals, and it is difficult to adapt RVB strains to cell culture $[9,12,14,23]$; as a result, the serological and molecular characterization of RVB strains remains limited. 
Table 1. Nucleotide and deduced amino acid sequence identity of the VP7 gene of the Brazilian strain BR62 and representatives of the different RVB G genotypes

\begin{tabular}{lll}
\hline \multirow{2}{*}{ Strain } & \multicolumn{2}{l}{ Identity with the BR62 strain, \% } \\
\cline { 2 - 3 } & nucleotide & amino acid \\
\hline RVB/Rat-hhp/USA/IDIR/1984/G1P[X] & 54.6 & 48.6 \\
RVB/Human-wt/BGD/Bang373/2000/G2P[X] & 64.3 & 64.1 \\
RVB/Cow-wt/JPN/Nemuro/1997/G3P[X] & 61.8 & 62.0 \\
RVB/Pig-wt/PB-70-H5/2007/G4P[X] & 71.2 & 75.9 \\
RVB/Cow-wt/IND/DB101/2001/G5P[X] & 63.8 & 62.0 \\
RVB/Pig-wt/USA/MN09-35/2009/G6P[X] & 64.7 & 61.4 \\
RVB/Pig-wt/JPN/PB-S15/2002/G7P[X] & 64.3 & 63.6 \\
RVB/Pig-wt/USA/CO09-12/2009/G8P[X] & 64.7 & 60.9 \\
RVB/Pig-wt/JPN/PB-68-E4/2007/G9P[X] & 62.2 & 60.9 \\
RVB/Pig-wt/USA/MO09-34/2009/G10P[X] & 64.8 & 64.1 \\
RVB/Pig-wt/JPN/PB-S5/2002/G11P[X] & 70.9 & 70.0 \\
RVB/Pig-wt/JPN/PB-S24-11/2002/G12P[X] & 69.1 & 68.9 \\
RVB/PigG13-wt/JPN/PB-23-44/2005/G13P[X] & 64.1 & 65.2 \\
RVB/Pig-wt/BRA/BR31/2012/G14P[X] & 67.9 & 67.3 \\
RVB/Pig-wt/JPN/PB-S49-2/2003/G15P[X] & 66.3 & 67.3 \\
RVB/Pig-wt/JPN/PB-68-C17/2007/G16P[X] & 66.1 & 64.1 \\
RVB/Pig-wt/USA/OK09-51/2009/G17P[X] & 66.6 & 62.5 \\
RVB/Pig-wt/USA/TX09-47/2009/G18P[X] & 66.6 & 64.1 \\
RVB/Pig-wt/IND/AN142129/2013/G19P[X] & 64.3 & 61.4 \\
RVB/Pig-wt/JPN/PB-S26-1/2002/G20P[X] & 64.8 & 60.4 \\
RVB/Pig-wt/IND/AN142530/2013/G21P[X] & 64.8 & 63.1 \\
RVB/Pig-wt/VNM/14177_18/2012/GXP[X] & 82.9 & 88.7 \\
& &
\end{tabular}

In 2009, Kuga et al. [9] proposed a classification system of G genotypes for RVB strains based on analyses of the VP7 genes of 38 porcine RVB strains. Using nt cutoffs of 67 and $76 \%$ (66 and 79\%, respectively, at the aa level), they proposed the creation of 5 genotypes divided into 12 clusters. Several years later, after phylogenetic analyses of 68 new RVB VP7 sequences had been performed, Marthaler et al. [12] proposed a modification to the cutoff values established by Kuga et al. [9]. Using an nt cutoff value of $80 \%$, they identified 20 RVB G genotypes. Additionally, using this nt cutoff value proposed by Marthaler et al. [12], Suzuki et al. [14] described a new RVB G genotype (G21) isolated from pigs in India.

In our study, the VP7 sequence of the BR62 RVB strain was compared with the VP7 sequences of representative strains for all 21 previously described $G$ genotypes. The BR62 RVB strain had the highest nt identity (71.2\%) with the porcine RVB strain PB-70-H5, which belongs to the G4 genotype. However, given that the VP7 gene sequence of the Brazilian strain BR62 did not share $\geq 80 \%$ nt identity with any of the VP7 gene sequences of RVB strains with previously identified genotypes, we propose the creation of G22, a new RVB G genotype.

In contrast, an analysis comparing the Brazilian BR62 strain and the unclassified Vietnamese RVB 14177_18 strain revealed an nt sequence identity of $82.9 \%$. This result led us to believe that strain 14177_18 and the Brazilian strain BR62 belong to the same genotype (G22). This assumption is supported by our nt-based phylogenetic tree, in which strains BR62 and 14177_18 are clustered together but placed in a different branch than strains belonging to the other $21 \mathrm{RVB}$ G genotypes. Moreover, this reasoning suggests that porcine RVB strains with genotypes distinct from previously described genotypes might be circulating in several countries, which may interfere with the control and prophylaxis of RV infections, as well as the zoonotic role of the virus. Therefore, screening for RVB should be actively incorporated into porcine RV studies, and RVB should receive increased research attention.

Regarding analyses of the deduced aa sequences for VP7 for the BR62 strain and other RVB strains, the BR62

Fig. 1. Phylogenetic trees constructed based on the nucleotide (a) and deduced amino acid (b) sequences of the VP7 gene from the porcine RVB strain BR62 described in this study, as well as representative strains for the 21 previously identified RVB genotypes. The scale bars indicate nt/aa substitutions per site. The bootstrap values are shown at branch nodes (values $<50 \%$ are not shown). The Brazilian porcine RVB strain is marked with a filled circle.

(For figure see next pages.)
Molinari/Alfieri/Alfieri 
a

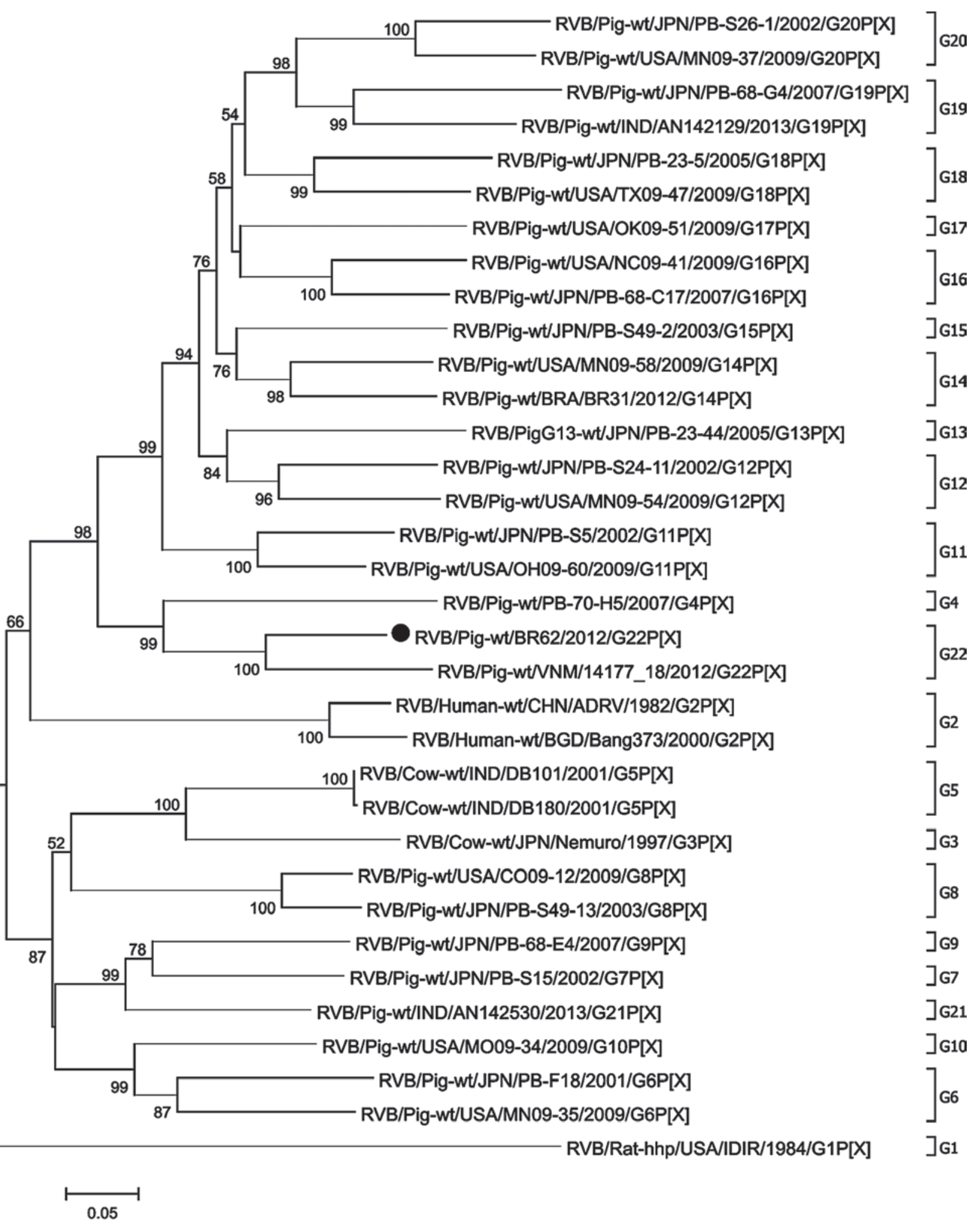


b

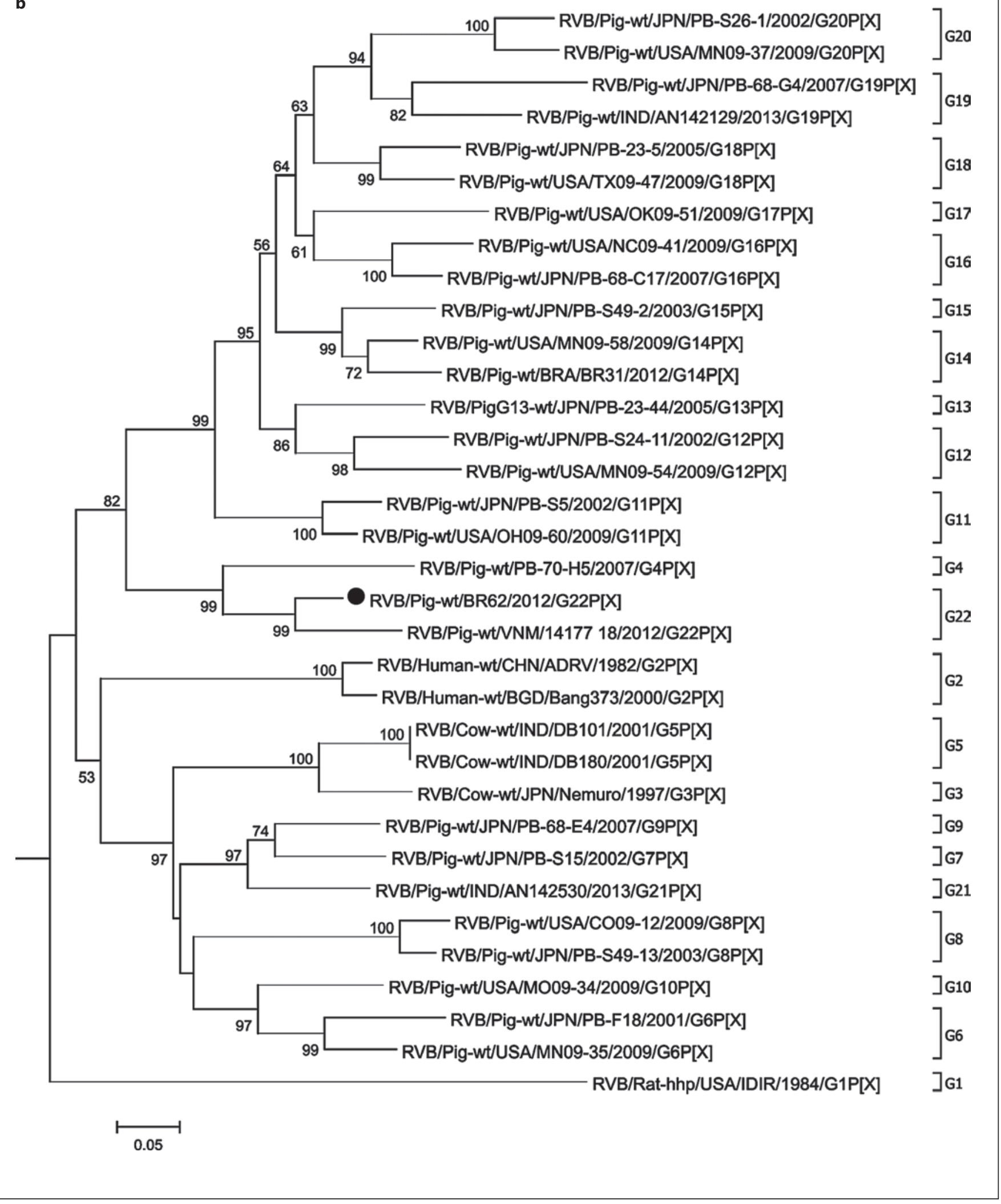


strain exhibited the highest $(88.7 \%)$ aa identity with the Vietnamese 14177_18 strain. According to Kuga et al. [9], aa cutoff values of 66 and $79 \%$ produced good correlations between $\mathrm{G}$ serotypes and $\mathrm{G}$ genotypes. Given this assumption, the Brazilian BR62 and Vietnamese 14177_18 strains could be classified as belonging to the same serotype. In contrast, Marthaler et al. [12] indicated that an aa cutoff value $>89 \%$ is appropriate for most RVB strains belonging to the same genotype, with certain exceptions (strains belonging to genotypes G6, G7, G12, and G16). Thus, two hypotheses seem reasonable: either the BR62 strain does not belong to the same serotype as the 14177_18 strain, or the new proposed G22 genotype is in the group of RVB genotypes with sufficient aa diversity such that the applicable cutoff threshold is lower than the suggested value of $89 \%$. If the second hypothesis is correct, the BR62 and 14177_18 strains would belong to the same serotype. The aa phylogenetic tree shows evidence that might support this conclusion; once again, the BR62 and 14177_18 strains are clustered together, but placed in a different branch than the other 21 RVB G genotypes. However, future serological assays should be performed to confirm these assumptions and provide more information about the viral immunology.

In conclusion, the genetic diversity observed among porcine RVB strains may be underestimated. In this study, two strains from different countries that belong to G22, a new RVB G genotype, could be identified. Although RVA infections are more prevalent and pathogenic than RVB infections, constant monitoring of RV infections is necessary to detect the emergence of new genotypes within various RV groups. Future studies of the epidemiology and molecular evolution of RVB genes should be developed to better understand and prevent RV infections. In addition, the idea that certain RV genotypes may exhibit a zoonotic potential and that animals can serve as reservoirs of such viruses reinforces the need for further studies.

\section{Acknowledgements}

This work was supported by the Brazilian Institutes $\mathrm{CNPq}$, CAPES, Finep, and Fundação Araucária (FAP/PR). A.F.A. and A.A.A. are recipients of $\mathrm{CNPq}$ fellowships.

\section{Statement of Ethics}

The study was submitted to the Ethics Committee on Animal Experiments of Universidade Estadual de Londrina and approved under the identification No. 11363.2015.16. All applicable international, national, and/or institutional guidelines for the care and use of animals were followed.

\section{Disclosure Statement}

All authors declare that they have no conflicts of interest.

\section{Funding Sources}

This study was funded by CNPq (grant No. 309.137/2013-6).

\section{Author Contributions}

B.L.D.M. developed this study as part of her postgraduate program under the guidance of A.A.A. and A.F.A. A.F.A. is the graduate student's co-advisor. She contributed to the design of the research as well as to corrections referring to the scientific manuscript. A.A.A. is the graduate student's advisor. He contributed to the design of the research as well as to corrections referring to the scientific manuscript.

\section{References}

1 Estes MK, Kapikian AZ: Rotaviruses; in Knipe DM, Howley PM, Griffin DE, Martin MA, Lamb RA (eds): Fields Virology, ed 5. Philadelphia, Lippincott, Williams \& Wilkins/ Wolters Kluwer, 2007, vol 2, pp 1917-1974.

2 Attoui H, Mertens PPC, Becnel J, Belaganahalli S, Bergoin M, et al: Family: Reoviridae; in King AMQ, Adams MJ, Carstens EB, Lefkowitz EJ (eds): Virus Taxonomy: Ninth Report of the ICTV. Amsterdam, Elsevier Academic Press, 2012, pp 541-637.
3 Matthijnssens J, Otto PH, Ciarlet M, Desselberger U, Van Ranst M, Johne R: VP6-sequence-based cutoff values as a criterion for rotavirus species demarcation. Arch Virol 2012;157:1177-1182.

4 Mihalov-Kovács E, Gellért Á, Marton S, Farkas SL, Fehér E, Oldal M, et al: Candidate new rotavirus species in sheltered dogs, Hungary. Emerg Infec Dis 2015;21:660-663.

5 Matthijnssens J, Martella V, Van Ranst M: Priority paper evaluation: genomic evolution, host-species barrier, reassortment and classification of rotaviruses. Future Virol 2010;5: 385-390.
6 Chasey D, Higgins RJ, Jeffrey M, Banks J: Atypical rotavirus and villous epithelial cell syncytia in piglets. J Comp Pathol 1989;100: 217-222.

7 Nagesha HS, Hum CP, Bridger JC, Holmes IH: Atypical rotaviruses in Australian pigs. Arch Virol 1988;102:91-98.

8 Fu ZF, Blackmore DK, Hampson DJ, Wilks CR: Epidemiology of typical and atypical rotavirus infections in New Zealand pigs. N Z Vet J 1989;37:102-106. 
9 Kuga K, Miyazaki A, Suzuki T, Takagi M, Hattori N, Katsuda K, et al: Genetic diversity and classification of the outer capsid glycoprotein VP7 of porcine group B rotaviruses. Arch Virol 2009;154:1785-1795.

10 Smitalova R, Rodak L, Smid B, Psikal I: Detection of nongroup A rotaviruses in faecal samples of pigs in the Czech Republic. Vet Med 2009;54:12-18.

11 Médici KC, Barry AF, Alfieri AF, Alfieri AA: Genetic analysis of the porcine group B rotavirus NSP2 gene from wild-type Brazilian strains. Braz J Med Biol Res 2010;43:13-16.

12 Marthaler D, Rossow K, Gramer M, Collins J, Goyal S, Tsunemitsu H, et al: Detection of substantial porcine group B rotavirus genetic diversity in the United States, resulting in a modified classification proposal for $\mathrm{G}$ genotypes. Virology 2012;433:85-96.

13 Lahon A, Ingle VC, Birade HS, Raut CG, Shobha D, Chitambar SD: Molecular characterization of group $\mathrm{B}$ rotavirus circulating in pigs from India: identification of a strain bearing a novel VP7 genotype, G21. Vet Microbiol 2014;174:342-352.
14 Suzuki T, Soma J, Kuga K, Miyazaki A, Tsunemitsu $\mathrm{H}$ : Sequence and phylogenetic analyses of nonstructural protein 2 genes of species B porcine rotaviruses detected in Japan during 2001-2009. Virus Res 2012;165:46-51.

15 Suzuki T, Kuga K, Miyazaki A, Tsunemitsu H Genetic divergence and classification of nonstructural protein 1 among porcine rotaviruses of species B. J Gen Virol 2011;92(pt 12): 2922-2929.

16 Suzuki T, Soma J, Miyazaki A, Tsunemitsu H: Phylogenetic analysis of nonstructural protein 5 (NSP5) gene sequences in porcine rotavirus B strains. Infect Genet Evol 2012;12: 1661-1668.

17 Marthaler D, Suzuki T, Rossow K, Culhane M, Collins J, Goyal S, et al: VP6 genetic diversity, reassortment, intragenic recombination and classification of rotavirus B in American and Japanese pigs. Vet Microbiol 2014;172: 359-366.

18 Molinari BLD, Alfieri AF, Alfieri AA: Genetic variability of VP6, VP7, VP4, and NSP4 genes of porcine rotavirus group $\mathrm{H}$ detected in $\mathrm{Bra}-$ zil. Virus Res 2015;197:48-53.

19 Molinari BLD, Possatti F, Lorenzetti E, Alfieri AF, Alfieri AA: Unusual outbreak of postweaning porcine diarrhea caused by single and mixed infections of rotavirus groups $\mathrm{A}, \mathrm{B}$, C, and H. Vet Microbiol 2016;193:125-132.
20 Phan MVT, Anh PH, Cuong NV, Munnink BBO, van der Hoek L, My PT, et al: Unbiased whole-genome deep sequencing of human and porcine stool samples reveals circulation of multiple groups of rotaviruses and a putative zoonotic infection. Virus Evol 2016;2: vew027.

21 Matthijnssens J, Ciarlet M, Heiman E, Arijs I, Delbeke T, McDonald SM, et al: Full genomebased classification of rotaviruses reveals a common origin between human Wa-like and porcine rotavirus strains and human DS-1like and bovine rotavirus strains. J Virol 2008; 82:3204-3219.

22 Aung MS, Nahar S, Aida S, Paul SK, Hossain MA, Ahmed S, et al: Distribution of two distinct rotavirus B (RVB) strains in the northcentral Bangladesh and evidence for reassortment event among human RVB revealed by whole genomic analysis. Infect Genet Evol 2017;47:77-86

23 Sanekata T, Kuwamoto Y, Akamatsu S, Sakon $\mathrm{N}$, Oseto $\mathrm{M}$, Taniguchi $\mathrm{K}$, et al: Isolation of group B porcine rotavirus in cell culture. J Clin Microbiol 1996;34:759-761. 